\title{
Predischarge transcutaneous bilirubin screening reduces readmission rate for hyperbilirubinaemia in diverse South African newborns: A randomised controlled trial
}

\author{
C Okwundu, ${ }^{1}$ MD , MPH, DCH, PhD; V K Bhutani, ${ }^{2}$ MD, FAAP; J Smith, ${ }^{3}$ MB ChB, PhD; T M Esterhuizen, ${ }^{1}$ BSc, MSc; C Wiysonge, ${ }^{1,4}$ MD, PhD \\ ${ }^{1}$ Division of Epidemiology and Biostatistics, Department of Global Health, Faculty of Medicine and Health Sciences, Stellenbosch University, \\ Cape Town, South Africa \\ ${ }^{2}$ Division of Neonatal and Developmental Medicine, Department of Pediatrics, Stanford School of Medicine, Lucile Packard Children's Hospital, \\ Palo Alto, CA, USA \\ ${ }^{3}$ Neonatology Unit, Department of Paediatrics and Child Health, Faculty of Medicine and Health Sciences, Stellenbosch University, Cape Town, South Africa \\ ${ }^{4}$ Cochrane South Africa, South African Medical Research Council, Cape Town, South Africa
}

Corresponding author: C Okwundu (charlesokwundu@gmail.com)

Background. In South Africa (SA), healthy term newborns are usually discharged $<72$ hours after delivery. Discharged babies remain at risk for severe hyperbilirubinaemia if it is not identified early. Hyperbilirubinaemia is an important cause of readmission, and also leads to neonatal mortality and morbidity. Use of transcutaneous bilirubin (TcB) screening before hospital discharge has been controversial. Objectives. To test the clinical benefits of TcB screening of healthy newborns before discharge for the outcomes of readmission for jaundice and severe hyperbilirubinaemia in a randomised controlled trial (RCT).

Methods. This was a RCT. We compared predischarge TcB screening with visual assessment (alone) for jaundice in apparently healthy newborns at a public tertiary hospital in Cape Town, SA. Patients or study participants were not involved in the study design and implementation.

Results. Of the 1858 infants, $63 \%$ were black, $35 \%$ of mixed race and $1 \%$ white. There was a significant reduction in the rate of readmission for jaundice (risk ratio (RR) 0.25 ; $95 \%$ confidence interval (CI) $0.14-0.46 ; p<0.0001$ ) and in the incidence of severe hyperbilirubinaemia (RR $0.27 ; 95 \%$ CI $0.08-0.97 ; p=0.05$ ) with the use of TcB screening compared with visual inspection.

Conclusions. Predischarge TcB screening is superior in identifying newborns at risk of severe hyperbilirubinaemia compared with visual inspection. We recommend that every newborn, regardless of skin pigmentation, should receive objective bilirubin screening before hospital discharge. Universal bilirubin screening in newborns could potentially reduce hyperbilirubinaemia-related morbidity and mortality.

S Afr Med J 2020;110(3):249-254. https://doi.org/10.7196/SAMJ.2020.v110i3.14186

Hyperbilirubinaemia is one of the common reasons for readmission of newborns in the first week after birth. ${ }^{[1,2]}$ Neonatal jaundice is usually benign and, in most instances, does not require intervention. However, up to $10 \%$ of term newborns ( $\geq 37$ weeks' gestational age) develop severe hyperbilirubinaemia that requires treatment, usually phototherapy and possibly exchange blood transfusion. ${ }^{[3]}$ Severe hyperbilirubinaemia is defined as a total serum bilirubin (TsB) concentration $>340 \mu \mathrm{mol} / \mathrm{L}(20 \mathrm{mg} / \mathrm{dL})$, and critical hyperbilirubinaemia as a TsB concentration $>425 \mu \mathrm{mol} / \mathrm{L}(25 \mathrm{mg} / \mathrm{dL})$ at any time during the first 28 days of life for late preterm (between 34 and 37 weeks' gestational age) and term newborns. ${ }^{[4]}$ Failure to identify newborns at risk of developing severe hyperbilirubinaemia can lead to severe posticteric neurological consequences, including athetoid cerebral palsy and a variety of lifelong manifestations of kernicterus. ${ }^{[5,6]}$

In many settings, term newborns are discharged from hospital 1 - 2 days after delivery, usually before hyperbilirubinaemia becomes clinically evident. Peak serum bilirubin levels usually occur on postnatal days $3-5$, when many newborns have already been discharged. The inability to identify and manage at-risk infants prior to hospital discharge in a timely manner has been cited as the major root cause of adverse outcomes of hyperbilirubinaemia. ${ }^{[7]}$ The burden of severe hyperbilirubinaemia continues to be high in low- and middle-income countries (LMICs). In many LMICs, severe or clinically significant neonatal hyperbilirubinaemia is not only a leading cause for hospital readmission in the first week of life, but also constitutes an important cause of neonatal mortality and morbidity. ${ }^{[8-10]}$ A report on the global burden of hyperbilirubinaemia suggests that sub-Saharan Africa and South Asia are the leading contributors to an estimated 1.1 million babies who develop severe hyperbilirubinaemia worldwide every year. ${ }^{[11]}$ In some cases, babies have levels that could lead to subsequent brain damage from kernicterus. ${ }^{[1-15]}$

Studies from African countries suggest that kernicterus is a leading cause of cerebral palsy. ${ }^{[12-14,16,17]}$ In SA, a retrospective study over 5 years of exchange transfusion for hyperbilirubinaemia in newborns showed that 6 of $26(23.0 \%)$ babies requiring exchange transfusion had signs of kernicterus. ${ }^{[18]}$ Findings from various observational studies conducted in North America suggest that universal bilirubin screening in newborns before discharge reduces the readmission rate for jaundice and the incidence of severe hyperbilirubinaemia. ${ }^{[19-22]}$ However, these findings have not been confirmed in any randomised controlled trial (RCT). ${ }^{[23]}$ Furthermore, there is no universal consensus on the routine screening for jaundice in all newborns using non-invasive transcutaneous bilirubin (TcB) screening or TsB measurement. ${ }^{[24-26]}$

The current SA guideline on the management of jaundice in the newborn does not make any recommendation on universal screening 
of all newborns with either TcB or TsB before hospital discharge. ${ }^{[27]}$ Similarly, in other African countries, there are no guidelines on universal screening of all newborns for risk of hyperbilirubinaemia. In many LMICs, newborns are assessed for jaundice by visual inspection and serum bilirubin is measured only in newborns who appear jaundiced or in some cases as part of a thyroid newbornscreening programme. ${ }^{[28]}$

In this RCT, we compared the use of a transcutaneous device with visual inspection to screen for hyperbilirubinaemia before hospital discharge in apparently healthy term and late preterm newborns ( $\geq 35$ weeks gestational age).

We compared the impact of TcB screening before hospital discharge with visual inspection for jaundice on readmission for hyperbilirubinaemia, and the incidence of severe hyperbilirubinaemia in a SA population of newborns.

\section{Methods \\ Study design and setting}

This was a randomised, controlled, unblinded trial that evaluated the effect of $\mathrm{TcB}$ screening in term newborns before discharge from hospital. The study was conducted at the well-baby nurseries at Tygerberg Hospital, Cape Town, SA. Tygerberg Hospital is the largest tertiary healthcare institution in Western Cape Province and has 308 paediatric beds and $\sim 7500$ deliveries per annum. According to current policy, well babies born at $\geq 35$ weeks' gestation and with a birthweight $\geq 1800 \mathrm{~g}$ are discharged home after 6 hours in the case of vaginal deliveries and 48 hours in the case of caesarean sections.

\section{Study procedures}

We screened all infants at the time of discharge for potential eligibility into the study using the predetermined inclusion and exclusion criteria. Those who were $\leq 72$ hours old, with a gestational age of at least 35 weeks and a birthweight of at least $1800 \mathrm{~g}$, were eligible for participation. The gestational age was determined by antenatal ultrasound scan, last menstrual period or postnatal foot length measured with a plastic Vernier's caliper for infants whose mothers were unbooked and did not have any prenatal dating scan. ${ }^{[2]}$

\section{Randomisation and blinding}

Infants who met all inclusion criteria were randomly assigned to either TcB screening for hyperbilirubinaemia or visual inspection for jaundice. A random allocation sequence was generated using a computer random sequence generator. Allocation assignments were recorded on sequentially numbered sheets of paper and placed in sealed opaque envelopes, maintained in a secure location and accessible only by the principal investigator (CO). The envelopes were opened sequentially for assignment by the research assistant after informed consent was obtained and the infants allocated to the group assigned in the envelope. The study group allocation was not blinded, as it is not possible to blind clinicians or parents to the type of screening provided due to the obvious nature of the intervention. The outcome assessors and statistician were blinded to the group allocation.

\section{Interventions}

\section{TcB screening group}

In this group, we measured $\mathrm{TcB}$ levels once using the JM-105 Jaundice Meter (Dräger Medical, UK), a portable, handheld, noninvasive $\mathrm{TcB}$ measuring device, at the time of enrolment. ${ }^{[30]}$ An average of 3 readings was plotted on Bhutani's hour-specific nomogram to determine the risk zone for hyperbilirubinaemia. Infants in this group were classified into the following groups: high risk, high intermediate risk, low intermediate risk and low risk (Fig. 1). ${ }^{[24]}$ For all newborns in the high-risk zone, we obtained a TsB. The need for phototherapy was based on the TsB measurement. Infants who met the threshold for phototherapy remained in hospital for this treatment. Babies in the high-intermediate-risk, lowintermediate-risk and low-risk groups were asked to attend follow-up at a primary healthcare centre closest to their home at 24 , 48 and 72 hours, respectively, after discharge home. We did not assess the timeliness of follow-up after discharge or the proportion of babies who went for follow-up.

\section{Visual inspection group}

Babies in this group were managed routinely according to the current standard of care. The infants were visually assessed for jaundice; the presence of tissue yellowness was assessed by blanching the skin over the glabella and sternum. The infants were evaluated by the admitting physician at the time of enrolment. Venous blood samples for TsB measurement were only collected in infants who were clinically jaundiced or in those of rhesus D-negative mothers, according to the hospital protocol. The need for phototherapy was determined based on the TsB result. Babies whose TsB value met the threshold for phototherapy according to the SA phototherapy guideline, were kept for such therapy before discharge home. ${ }^{[27]}$ Babies who did not meet the threshold for phototherapy and who were not visibly jaundiced at the time of discharge, were managed routinely. The mothers were asked to return with their newborns for followup assessments at the primary healthcare facility closest to their home within 2 days of hospital discharge.

\section{Sample size}

To the best of our knowledge, this is the first published study that assessed the rate of readmission for hyperbilirubinaemia in SA newborns. We assumed a baseline readmission rate for phototherapy of $4.8 \%$ from the literature and an unpublished local study (Ndayisaba, 2014) at Tygerberg Hospital on newborn readmission for hyperbilirubinaemia. ${ }^{[31-33]}$ We estimated

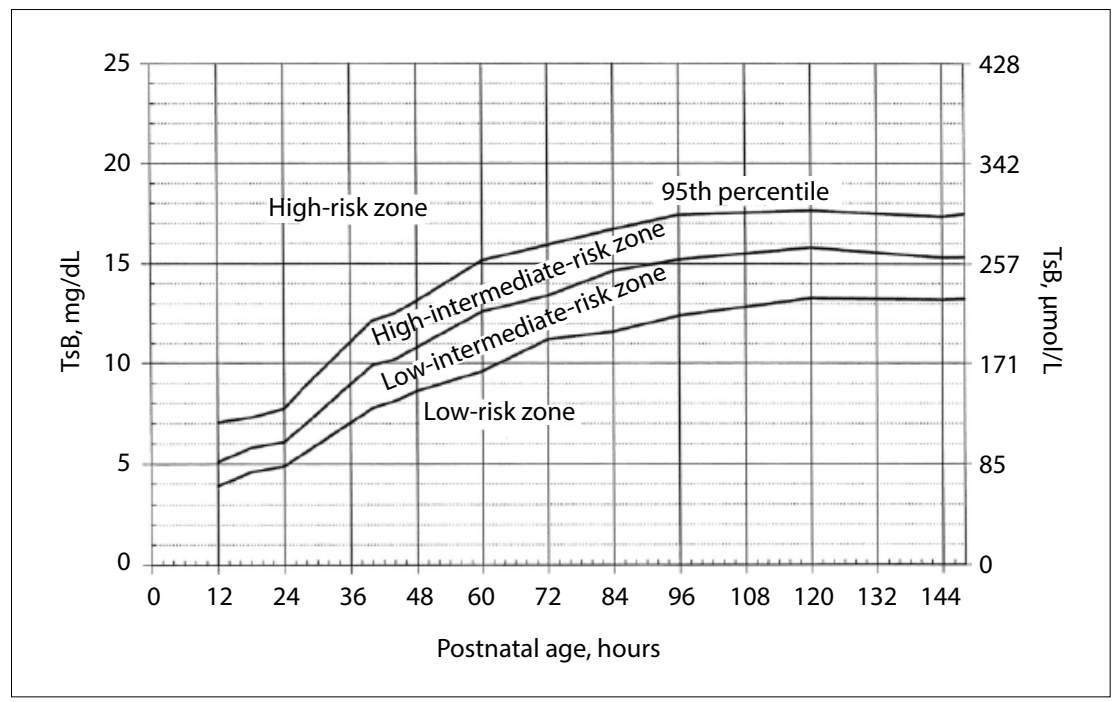

Fig. 1. Bhutani nomogram, with permission from Dr Bhutani. ${ }^{[2]}$ (TsB = total serum bilirubin.) 
that a minimum of 1858 participants would give us $80 \%$ power to detect a $50 \%$ reduction in the rate of readmission with the use of $\mathrm{TcB}$ screening at a $5 \%$ level of significance.

\section{Study outcomes}

The primary endpoint was readmission for jaundice that required phototherapy or exchange transfusion. For the primary outcome, we compared the $\mathrm{TcB}$ group with the visual inspection group regarding the proportion of readmissions for hyperbilirubinaemia that required phototherapy or exchange transfusion. For every infant who was readmitted, we ascertained whether they met the threshold for either phototherapy or exchange transfusion according to the SA phototherapy guideline, based on the TsB result at the time of readmission. ${ }^{[27]}$

The secondary endpoints were: phototherapy before hospital discharge, incidence of severe hyperbilirubinaemia, critical hyperbilirubinaemia or exchange transfusions and duration of hospital stay for those who were readmitted. The rate of phototherapy before discharge was measured during hospitalisation for the birth. Other outcomes (readmission for phototherapy, incidence of severe hyperbilirubinaemia and duration of hospital stay) were determined electronically through the database and also by obtaining the infants' files and electronic patient record transcripts from the hospital or primary healthcare centre where the baby was readmitted. We obtained all TsB values during the first month of life for newborns who were readmitted. For each readmission, we confirmed the name of the hospital or primary healthcare centre where the baby was readmitted, length of stay, treatment received (phototherapy or exchange transfusion) and highest TsB level during the readmission period.

\section{Statistical analysis}

We collected study data using paper-based data collection forms and then transferred to the REDCap (Research Electronic Data Capture) database. Statistical analyses were done with the use of Stata 14 statistical software (StataCorp., USA).

We performed an intention-to-treat analysis. Differences according to the group allocation for continuous variables were tested with the use of unpaired $t$-tests with equal variances and Wilcoxon rank-sum tests. The Pearson $\chi^{2}$ test was used to test for differences between the two groups for categorical variables. Fisher's exact test was used to compare the rate of phototherapy before hospital discharge, readmission for hyperbilirubinaemia and incidence of severe hyperbilirubinaemia. Effect sizes were reported as risk ratios (RRs) with $95 \%$ confidence intervals (CIs). We also adjusted for any plausible baseline-confounding variable (e.g. history of jaundice in a previous sibling) by performing Mantel-Haenszel stratification.

\section{Patient involvement}

Patients or study participants were not involved in the design and implementation of the study.

\section{Ethical approval}

We obtained ethical approval from the Health Research Ethics Committee of the Faculty of Medicine and Health Sciences, Stellenbosch University (ref. no. N14/03/025). Written informed consent was obtained from the mothers for all participants before enrolment into the study. The trial was registered on clinicaltrials.gov (https://clinicaltrials.gov/ ct2/show/NCT02613676).

\section{Results}

Participant recruitment and follow-up We enrolled a cohort of 1858 late preterm and term infants born between August 2015 and October 2016. A total of 1910 mothers were approached for participation. Two mothers declined, and 50 were excluded because they did not meet our inclusion criteria (Fig. 2). A total of 929 infants were enrolled to each arm. All infants were screened according to the study arm to which they were allocated.

\section{Participant characteristics}

The baseline characteristics and demographics of enrolled infants and their mothers are shown in Table 1. There were no significant differences between the TcB screening arm and the visual inspection arm, except for the history of jaundice in a previous sibling, which was significantly higher in the TcB group.

\section{Outcome measures}

Readmission for jaundice

There was a total of 65 (3.5\%) jaundicerelated readmissions. We obtained the TsB results for these infants to determine if they met the threshold for phototherapy on readmission. We could not obtain the TsB results of 5 ( 3 in the visual inspection arm and 2 in the TcB arm) infants who were readmitted after discharge, and could not determine the appropriateness of their readmission.

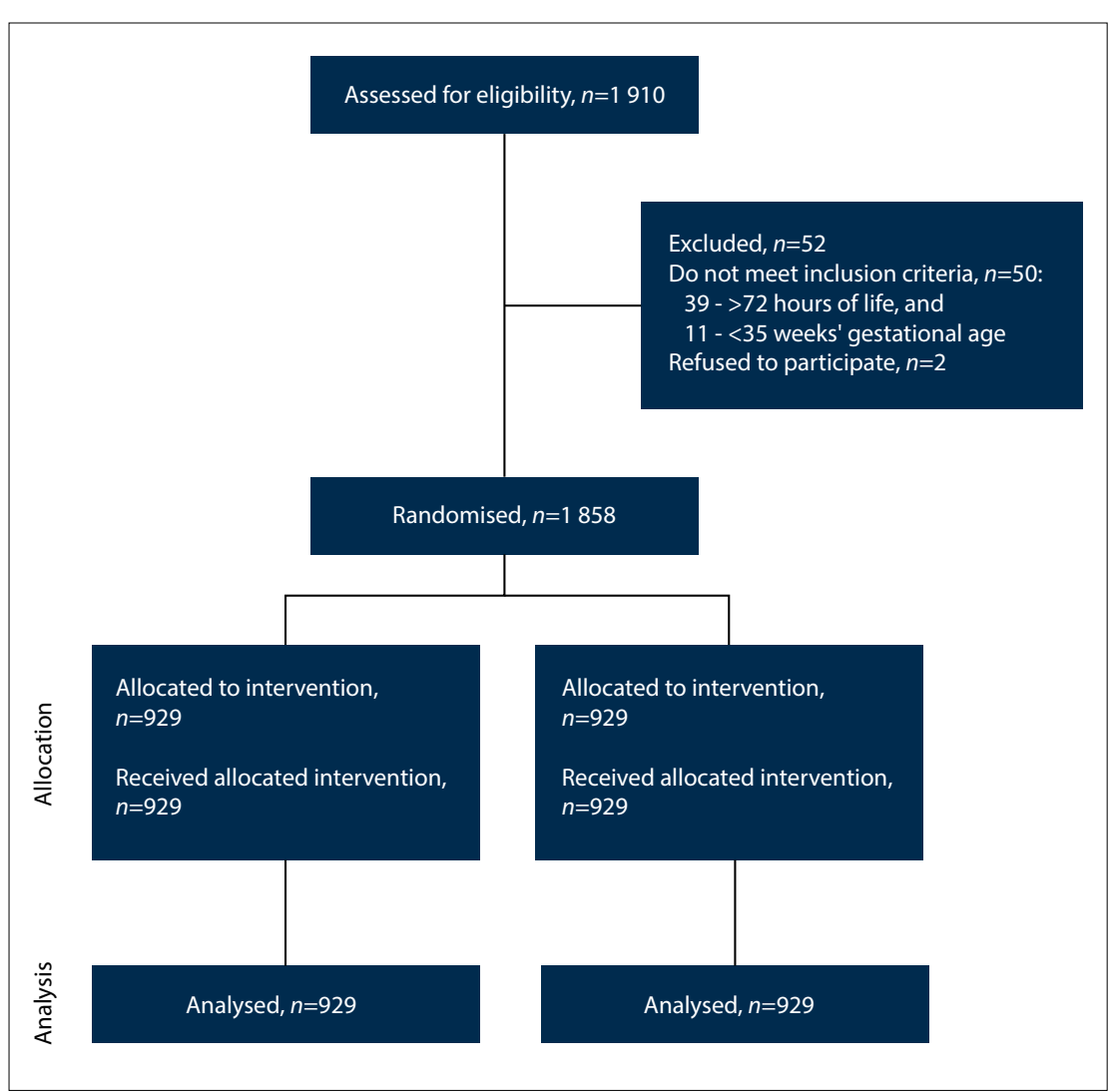

Fig. 2. Participant study flow diagram. 


\begin{tabular}{|c|c|c|c|}
\hline Variable & TcB screening $(N=929), n(\%)$ & Visual inspection $(N=929), n(\%)$ & $p$-value \\
\hline Gestational age (weeks), mean (SD) & $38.4(1.60)$ & $38.3(1.60)$ & 0.332 \\
\hline$<37$ weeks' gestation & $123(13.2)$ & $144(15.5)$ & 0.232 \\
\hline$\geq 37$ weeks' gestation & $806(86.8)$ & $785(84.5)$ & 0.212 \\
\hline Birthweight (g), mean (SD) & $3128(575)$ & $3118(566)$ & 0.709 \\
\hline IUGR & $106(12.70)$ & $118(11.41)$ & 0.393 \\
\hline Male & $485(52.2)$ & $480(51.67)$ & 0.816 \\
\hline \multicolumn{4}{|l|}{ Race } \\
\hline Black & $590(63.5)$ & $585(63.0)$ & 0.320 \\
\hline Mixed & $319(34.3)$ & $334(36.0)$ & 0.320 \\
\hline White & $15(1.3)$ & $8(0.9)$ & 0.320 \\
\hline Other & $3(0.3)$ & $2(0.2)$ & 0.320 \\
\hline Breastfeeding & $870(93.65)$ & $893(96.12)$ & 0.052 \\
\hline Cephalohaematoma & $1(0.11)$ & $2(0.22)$ & 0.056 \\
\hline Previous sibling with jaundice & $72(7.75)$ & $50(5.38)$ & 0.039 \\
\hline Age at time of assessment, hours & 31.1 & 31.9 & 0.283 \\
\hline \multicolumn{4}{|l|}{ Mode of delivery } \\
\hline NVD & $492(52.96)$ & $510(54.90)$ & 0.287 \\
\hline Caesarean section & $425(45.75)$ & $413(44.46)$ & 0.287 \\
\hline Vacuum & $12(1.29)$ & $6(0.65)$ & 0.287 \\
\hline \multicolumn{4}{|l|}{ Mother's Rh status } \\
\hline Negative & $16(1.72)$ & $14(1.51)$ & 0.927 \\
\hline Positive & $871(93.76)$ & $874(94.08)$ & 0.927 \\
\hline Unknown & $42(4.52)$ & $41(4.41)$ & 0.927 \\
\hline \multicolumn{4}{|l|}{ Mother's blood group } \\
\hline A & $31(3.34)$ & $25(2.69)$ & 0.666 \\
\hline $\mathrm{AB}$ & $7(0.75)$ & $3(0.32)$ & 0.666 \\
\hline $\mathrm{B}$ & $16(1.72)$ & $16(1.72)$ & 0.666 \\
\hline $\mathrm{O}$ & $37(3.98)$ & 35 (3.77) & 0.666 \\
\hline
\end{tabular}

Thirteen of 929 (1.4\%) infants were readmitted in the TcB arm compared with 52 of 929 (5.6\%) infants in the visual inspection arm (RR 0.25; 95\% CI $0.14-0.46 ; p<0.0001$ ). (Number needed to treat $(\mathrm{NNT})=24 ; 95 \%$ CI 17 - 40.) Among all infants who were readmitted, there were 12 appropriate readmissions in the TcB group and 48 appropriate readmissions in the visual inspection group. As there was a randomisation imbalance in the groups regarding a previous baby with jaundice, adjustment was done by stratified analysis. The Mantel-Haenszel adjusted RR was 0.25 (95\% CI 0.14 0.46 ), indicating that after adjustment for having a previous baby with jaundice, there was still a significantly protective effect of TcB screening on readmission for jaundice.

\section{Phototherapy before discharge}

The rate of phototherapy before hospital discharge was significantly higher in the TcB group than in the visual inspection group. A total of 48 (5.2\%) infants received phototherapy before hospital discharge compared with $18(1.9 \%)$ in the visual inspection group (RR 2.66; $95 \%$ CI 1.56 - 4.54; $p=0.0002$ ). In the TcB arm, 83 (9\%) babies had predischarge $\mathrm{TcB}$ values in the high-risk zone ( $\geq 95$ th percentile); of these, 39 (47\%) had TsB levels at which phototherapy was recommended according to the SA phototherapy guideline. A total of 131 (14\%), 173 (19\%) and 542 (58\%) infants had predischarge TcB measurements in the high-intermediate, low-intermediate and low-risk zones, respectively.

\section{Incidence of severe hyperbilirubinaemia}

A total of $14(0.75 \%)$ infants had severe hyperbilirubinaemia at the time of readmission -3 in the $\mathrm{TcB}$ arm and 11 in the visual inspection $\mathrm{arm}$. The rate of severe hyperbilirubinaemia was significantly lower with the use of TcB screening (RR 0.27; 95\% CI 0.08 - 0.970; $p=0.05$ ).

\section{Rate of exchange transfusion}

Among those who were readmitted, all infants in the TcB group required phototherapy and none met the threshold for exchange transfusion. Two infants in the visual inspection group who were readmitted required exchange transfusions. This difference was not statistically significant.

\section{Bilirubin-induced neurological dysfunction}

There was 1 infant in the visual inspection group who had clinical signs that could suggest bilirubin-induced neurological dysfunction. The baby was born at 36 weeks' gestational age with a birth weight of $2440 \mathrm{~g}$, discharged home at 72 hours after delivery, and readmitted 2 weeks later owing to prolong ed neonatal jaundice. On readmission, the baby was noted to have lost about $18 \%$ of the birth weight, had a high-pitched cry and a TsB of $459 \mu \mathrm{mol} / \mathrm{L}$. No magnetic resonance imaging (MRI) of the brain was done and the long-term neurological outcome for this infant is unknown. There was no documented case of infants showing neurological dysfunction in the TcB arm. 


\section{TsB before discharge}

A total of 87 (9\%) infants required TsB before discharge in the TcB group compared with $70(7.5 \%)$ in the visual inspection group (RR 1.24; 95\% CI $0.91-1.68$; $p=0.156$ ). The difference was not statistically significant.

\section{Duration of hospital stay}

The median duration of hospital stay during the readmission period was similar in both groups. Median duration of hospital stay in the visual inspection group was 3 (interquartile range (IQR) 2 - 4) days compared with 3 (IQR 2 - 3) days in the TcB group. The Wilcoxon rank-sum (Mann-Whitney) test did not demonstrate any statistically significant difference between arms $(p=0.216)$.

\section{Discussion}

In this RCT involving indigenous South African newborns recruited from a tertiary hospital in SA, we provide evidence for the effect of predischarge $\mathrm{TcB}$ screening on readmission for jaundice and incidence of severe hyperbilirubinaemia. To our knowledge, this RCT is the first to evaluate the impact of TcB screening in a South African population of newborns. We demonstrated that TcB screening for hyperbilirubinaemia was superior in identifying apparently healthy newborns requiring phototherapy before hospital discharge compared with visual inspection. Earlier identification of hyperbilirubinaemia combined with treatment with phototherapy during the initial hospitalisation for the birth led to a reduction in readmission rate with the use of $\mathrm{TcB}$ screening.

Unexpected readmission of a newborn after discharge is an indicator of inadequate assessment of the newborn's readiness for discharge. For our study, we selected the readmission rate as a primary outcome. Although this index is often viewed from the perspective of healthcare cost, epidemiological burden and societal burden of trust between healthcare provider and the family, it can have a direct personal disruption in the maternal and newborn experience. ${ }^{[34-37]}$

In 2004, the American Academy of Pediatrics (AAP) recommended a patient-friendly systems approach, i.e. that every newborn should be assessed for the risk of developing severe hyperbilirubinaemia by using predischarge TsB or TcB measurements, a policy also endorsed by the Canadian Paediatric Society (CPS).$^{[4,24]}$ After implementation of the AAP and CPS guidelines, many studies in the USA and Canada have demonstrated that universal $\mathrm{TcB}$ or $\mathrm{TsB}$ reduced jaundice readmissions and led to a reduction in the incidence of severe hyperbilirubinaemia. ${ }^{[20-22,32,38,39]}$ Our study not only validates the finding from these studies, but also provides evidence that earlier identification of hyperbilirubinaemia with $\mathrm{TcB}$ screening and treatment with phototherapy is associated with a reduction in hospital readmission rates for hyperbilirubinaemia and in the incidence of severe hyperbilirubinaemia in an indigenous population of South African newborns.

Other studies from LMICs have evaluated the correlation of $\mathrm{TcB}$ compared with TsB measurement in newborns with different skin pigmentation. These have shown a good correlation, and that implementation of TcB screening is feasible in LMICs. ${ }^{[40-47]}$ However, because of varying access to healthcare and differential risk in the polycultural community of LMICs, there are limited - if any national policy outcome studies. Therefore, we could not identify any studies from LMICs that evaluated the impact of TcB screening on readmission rate or incidence of severe hyperbilirubinaemia.

\section{Study limitations}

Our study has some important limitations. It did not evaluate the impact of TcB screening on reducing the incidence of kernicterus, which would require a very large study and longer follow-up period. In our study, only 1 infant in the visual inspection arm had a clinical sign of probable kernicterus. Blood group typing and the direct Coombs' test for blood group incompatibility were not routinely done on all newborns. Therefore, we could not assess whether there was any difference in the rate of blood group incompatibility between the two study arms. It was difficult to determine if the highest TsB measurement was recorded prior to phototherapy initiation. In some facilities where babies were readmitted, phototherapy was initiated before a blood sample for TsB was collected. Therefore, the incidence of severe hyperbilirubinaemia in the study population could be higher than that reported. Blood for TsB measurement was ordered at the discretion of the admitting physician. The outcome would possibly have been different if we limited blood sampling only to infants whose TcB value was $>95$ th percentile for their age. This study was conducted in a tertiary facility where some mothers were kept longer because of other medical reasons and consequently the babies were also kept longer together with their mothers. This increased the length of stay for some babies, which could lead to a reduction in readmission because of delayed discharge from the initial hospitalisation for the birth. Therefore, the readmission rates reported are likely to be higher in the primary healthcare setting in SA, where mothers without any complications after delivery are typically discharged within 12 hours of delivery. However, despite these limitations, we are confident about the findings, because of the unique design of the study compared with other non-randomised studies that have evaluated similar outcomes. ${ }^{[20-22,32,38,39]}$ We have provided evidence from a randomised controlled study. We used a proper method of randomisation and allocation concealment, with sealed opaque envelopes. Even though this method of concealment of allocation is prone to deliberate tampering, the investigators ensured that the envelopes were only opened at the time of enrolment. The study participants were similar with regard to the baseline characteristics. Although the study personnel and participants were not blinded to the treatment allocation, the outcome assessors and statistician were blinded. Therefore, we are confident that the reduction in hospital readmission rate and in the incidence of severe hyperbilirubinaemia was as a result of the TcB screening strategy in the treatment group.

\section{Conclusions}

TcB screening with risk stratification using the Bhutani nomogram in newborn infants at risk of severe hyperbilirubinaemia can help to reduce readmissions for jaundice and the incidence of severe hyperbilirubinaemia. Our study further confirms that visual inspection for jaundice is not reliable to assess the presence or absence of hyperbilirubinaemia in newborns. Universal TcB screening in all newborns before hospital discharge in SA and other African countries will be an important step towards reducing the burden and consequences of severe hyperbilirubinaemia. However, the high cost of the current TcB devices could be a barrier to implementation of universal TcB screening in many LMICs. Currently, a TcB device could cost between ZAR50 000 and ZAR100 000. However, the use of $\mathrm{TcB}$ to screen for hyperbilirubinaemia in newborns has been shown to be good value for money. ${ }^{[48]}$ Therefore, nationally driven public-private solutions are warranted, such that healthcare during 
the first week after a normal pregnancy is equitable. Industry and $\mathrm{TcB}$ manufacturers now have the moral responsibility to make over-priced transcutaneous devices safe, affordable and accessible. Reductions in the cost of readmission, the cost of more intensive treatment, and the family's integrity are real and need to be realised for all newborns worldwide.

Declaration. This study was done as part of CO's PhD thesis, but the publication was not a requirement for the degree.

Acknowledgements. Dräger South Africa donated the TcB device used in the study.

Author contributions. CO: conceptualised and designed the study, designed the data collection tools, co-ordinated and supervised data collection at the study site, drafted the initial manuscript and approved the final manuscript; VKB, CW, JS: provided input regarding the methods and content knowledge, critically reviewed the manuscript and approved the final manuscript; TME: conducted the statistical analysis, reviewed the manuscript and approved the final manuscript.

Funding. This study was funded by the South African Medical Research Council. The study sponsors were not involved in any aspect of the study. Conflicts of interest. None.

Availability of data and materials. The datasets used and/or analysed during the study are available from the corresponding author on reasonable request.

1. Brown AK, Damus K, Kim MH, et al. Factors relating to readmission of term and near-term neonates in the first two weeks of life. J Perinat Med 1999;27(4):263-275. https://doi.org/10.1515/JPM.1999.037

2. Liu S, Wen SW, McMillan D, Trouton K, Fowler D, McCourt C. Increased neonatal readmissio rate associated with decreased length of hospital stay at birth in Canada. Can J Public Health 2000;91(1):46-50.

3. Maisels MI. Managing the jaundiced newborn: A persistent challenge. Can Med Ass J 2015;187(5):335-343. https://doi.org/10.1503/cmaj.122117

4. Canadian Paediatric Society. Guidelines for detection, management and prevention of hyperbilirubinemia in term and late preterm newborn infants ( 35 or more weeks' gestation). Paediatr Child Health in term and late preterm newborn infants $(35$ or m

5. Bhutani VK, Wong R. Bilirubin-induced neurologic dysfunction (BIND). Semin Fetal Neonatal Med 2015;20(1):1. https://doi.org/10.1016/.siny.2014.12.010

2015;20(1):1. https://doi.org/10.1016/j.siny.2014.12.010
6. Sgro M, Campbell D, Shah V. Incidence and causes of severe neonatal hyperbilirubinemia in Canada. 6. Sgro M, Campbell D, Shah V. Incidence and causes of severe neonatal hy
Can Med Ass J 2006;175(6):587-590. https://doi.org/10.1503/cmaj.060328

7. Johnson L, Bhutani VK, Karp K, Sivieri EM, Shapiro SM. Clinical report from the pilot USA Kernicterus Registry (1992 to 2004). J Perinatol 2009;29(Suppl 1):S25-S45. https://doi.org/10.1038/jp.2008.211

8. Greco C, Arnolda G, Boo NY, et al. Neonatal jaundice in low- and middle-income countries: Lessons and future directions from the 2015 Don Ostrow Trieste Yellow Retreat. Neonatology 2016;110(3):172-180 https://doi.org/10.1159/000445708

9. Olusanya BO, Ogunlesi TA, Slusher TM. Why is kernicterus still a major cause of death and disability in low-income and middle-income countries? Arch Dis Child 2014;99(12):1117-1121. https://doi.org/ 10.1136/archdischild-2013-305506

10. Ugwu RO, Eneh AU, Oruamabo RS. Blood transfusion therapy in neonates admitted into the special care baby unit (SCBU) of University of Port Harcourt Teaching Hospital, Port Harcourt. Niger J Med 2006;15(4):401-405. https://doi.org/10.4314/njm.v15i4.37253

11. Bhutani VK, Zipursky A, Blencowe H, et al. Neonatal hyperbilirubinemia and rhesus disease of the newborn: Incidence and impairment estimates for 2010 at regional and global levels. Pediatr Res
nestatis newborn: Incidence and impairment estimates for 2010 at

12. Donald KA, Samia P, Kakooza-Mwesige A, Bearden D. Pediatric cerebral palsy in Africa: A systematic review. Semin Pediatr Neurol 2014;21(1):30-35. https://doi.org/10.1016/j.spen.2014.01.001

13. Olusanya BO, Osibanjo FB, Mabogunje CA, Slusher TM, Olowe SA. The burden and management of neonatal jaundice in Nigeria: A scoping review of the literature. Niger J Clin Pract 2016;19(1):1-17. https://doi.org/10.4103/1119-3077.173703

14. Iskander I, Gamaleldin R, El Houchi S, et al. Serum bilirubin and bilirubin/albumin ratio as predictors of bilirubin encephalopathy. Pediatrics 2014;134(5):e1330-e1339. https://doi.org/10.4103/1119 3077.173703

15. Mwaniki MK, Gatakaa HW, Mturi FN, et al. An increase in the burden of neonatal admissions to a rural district hospital in Kenya over 19 years. BMC Public Health 2010;10:591. https://doi.org/10.1186/14712458-10-591
16. Nottidge VA, Okogbo ME. Cerebral palsy in Ibadan, Nigeria. Dev Med Child Neurol 1991;33(3):241-245. 7. Belonwu RO, Gwarzo GD, Adeleke SI. Cerebral palsy in Kano, Nigeria - a review. Niger J Med 2009;18(2):186-189. https://doi.org/10.4314/njm.v18i2.45062 18. Ballot DE, Rugamba G. Exchange transfusion for neonatal hyperbilirubinemia in Johannesburg, South

19. Wainer S, Parmar SM, Allegro D, Rabi Y, Lyon ME. Impact of a transcutaneous bilirubinometry program on resource utilization and severe hyperbilirubinemia. Pediatrics 2012;129(1):77-86. https:// program on resource utilization

20. Mah MP, Clark SL, Akhigbe E, et al. Reduction of severe hyperbilirubinemia after institution of predischarge bilirubin screening. Pediatrics 2010;125(5):el143-el148. https://doi.org/10.1542/ peds.2009-1412

21. Kuzniewicz MW, Escobar GJ, Newman TB. Impact of universal bilirubin screening on severe hyperbilirubinemia and phototherapy use. Pediatrics 2009;124(4):1031-1039. https://doi.org/10.1542/ peds.2008-2980

22. Eggert LD, Wiedmeier SE, Wilson J, Christensen RD. The effect of instituting a prehospital-discharge newborn bilirubin screening program in an 18-hospital health system. Pediatrics 2006;117(5):e855-e862. https://doi.org/10.1542/peds.2005-1338

23. Sharma A. Effectiveness of a pre-discharge bilirubin screening in high-risk neonates - is the evidence robust enough? Indian Pediatr 2013;50(2):248. https://doi.org/10.1007/s13312-013-0046-8

24. American Academy of Pediatrics Subcommittee on Hyperbilirubinemia. Management of hyperbilirubinemia in the newborn infant 35 or more weeks of gestation. Pediatrics 2004;114(1):297-316. https://doi.org/10.1542/peds.114.1.297

25. US Preventive Services Task Force Recommendation Statement. Screening of infants for hyperbilirubinemia to prevent chronic bilirubin encephalopathy. Pediatrics 2009;124(4):1172-1177. https://doi.org/10.1542/peds.2009-012

26. Rennie J, Burman-Roy S, Murphy MS, Guideline Development Group. Neonatal jaundice: Summary of NICE guidance. BMJ 2010;340:c2409. https://doi.org/10.1136/bmj.c2409

27. Horn AR, Kirsten GF, Kroon SM, et al. Phototherapy and exchange transfusion for neonatal hyperbilirubinaemia: Neonatal academic hospitals' consensus guidelines for South African hospitals and primary care facilities. S Afr Med J 2006;96(9):819-824. https://doi.org/10.7196/SAMJ.1256

28. Basheer H, Makhlouf M, El Halawany F, Fahmy N, Iskander I. Screening for neonatal jaundice in El Galaa Teaching Hospital: An Egyptian maternity hospital - can the model be replicated? J Clin Neonatol 2017;6:128-133.

29. Wyk LV, Smith J. Postnatal foot length to determine gestational age: A pilot study. J Trop Pediatr 2016;62(2):144-151. https://doi.org/10.1093/tropej/fmv093

30. Yasuda S, Itoh S, Isobe K, et al. New transcutaneous jaundice device with two optical paths. J Perinat Med 2003;31(1):81-88. https://doi.org/10.1515/JPM.2003.012

31. VanderWal B, Kyle C. Decreasing newborn readmissions for hyperbilirubinemia. J Obstet Gynecol Neonatal Nurs 2015;44(1):S25. https://doi.org/10.1111/1552-6909.12698

32. Alkalay AL, Bresee CJ, Simmons CF. Decreased neonatal jaundice readmission rate after implementing hyperbilirubinemia guidelines and universal screening for bilirubin. Clin Pediatr (Phila) 2010;49(9):830-833. https://doi.org/10.1177/0009922810363728

33. Escobar GJ, Greene JD, Hulac P, et al. Rehospitalisation after birth hospitalisation: Patterns among infants of all gestations. Arch Dis Child 2005;90(2):125-131. https:// doi.org/10.1136/adc.2003.039974

34. Bhutani VK, Johnson LH, Jeffrey Maisels M, et al. Kernicterus: Epidemiological strategies for its prevention through systems-based approaches. J Perinatol 2004;24(10):650-662. https://doi.org/10.1038/ sj.jp. 7211152

35. Meara E, Kotagal UR, Atherton HD, Lieu TA. Impact of early newborn discharge legislation and early follow-up visits on infant outcomes in a state Medicaid population. Pediatrics 2004:113(6):1619-1627. https://doi.org/10.1542/peds.113.6.1619

36. Sheridan SE. Parents of infants and children with kernicterus. J Perinatol 2005;25(4):227-228. https:// doi.org/10.1038/sj.jp.7211244

37. American Academy of Pediatrics. Committee on Fetus and Newborn. Hospital stay for healthy term newborns. Pediatrics 2010;125(2):405-409. https://doi.org/10.1542/peds.2009-3119

38. Darling EK, Ramsay T, Sprague AE, Walker MC, Guttmann A. Universal bilirubin screening and health care utilization. Pediatrics 2014;134(4):e1017-e10124. https://doi.org/10.1542/peds.2014-1146

39. Petersen JR, Okorodudu AO, Mohammad AA, Fernando A, Shattuck KE. Association of transcutaneous bilirubin testing in hospital with decreased readmission rate for hyperbilirubinemia. Clin Chem 2005;51(3):540-544. https://doi.org/10.1373/clinchem.2004.037804

40. Slusher TM, Angyo IA, Bode-Thomas F, et al. Transcutaneous bilirubin measurements and serum total bilirubin levels in indigenous African infants. Pediatrics 2004;113(6):1636-1641. https://doi.org/10.1542/ peds.113.6.1636

41. Akahira-Azuma $M$, Yonemoto $N$, Ganzorig $B$, et al Validation of a transcutaneous bilirubin meter in Mongolian neonates: Comparison with total serum bilirubin. BMC Pediatr 2013;13:151.

42. Bhutta ZA, Yusuf K. Transcutaneous bilirubinometry in Pakistani newborns: A preliminary report. I Pak Med Assoc 1991;41(7):155-156

43. Rylance S, Yan J, Molyneux E. Can transcutaneous bilirubinometry safely guide phototherapy treatment of neonatal jaundice in Malawi? Paediatr Int Child Health 2014;34(2):101-107. https://doi.org/10.1179/ $2046905513 Y .0000000050$

44. Lam TS, Tsui KL, Kam CW. Evaluation of a point-of-care transcutaneous bilirubinometer in Chinese neonates at an accident and emergency department. Hong Kong Med J 2008;14(5):356-360.

45. Janjindamai W, Tansantiwong T. Accuracy of transcutaneous bilirubinometer estimates using BiliCheck in Thai neonates. J Med Assoc Thai 2005;88(2):187-190.

46. Olusanya BO, Emokpae AA. Use of transcutaneous bilirubin to determine the need for phototherapy in resource-limited settings. Neonatology 2017;111(4):324-330. https://doi.org/10.1159/000452788

47. Morgan MC, Kumar GS, Kaiser SV, Seetharam S, Ruel TD. Implementation of a neonatal transcutaneous bilirubin screening programme in rural India. Paediatr Int Child Health 2016;36(2):122-126. https://doi. org/10.1179/2046905515Y.0000000013

48. Dowsett L, Soril L, Clement F, et al.Transcutaneous bilirubinometers for newborn hyperbilirubinemia. A health technology assessment. 2017. https://www2.gov.bc.ca/assets/gov/health/about-bc-s-health-caresystem/heath-care-partner/health-authorities/bc-health-technology-assessments/bilirubinometers-fornewborn-hyperbilirubinemia.pdf (accessed 30 August 2018).

Accepted 13 August 2019. 